\title{
Microstructural Features of Current Resin Composite Materials
}

\author{
Jack L. Ferracane • Carmem S. Pfeifer • \\ Thomas J. Hilton
}

Published online: 14 September 2014

(C) Springer International Publishing AG 2014

\begin{abstract}
Dental resin composites have become the primary choice of most practitioners for the direct restoration of teeth owing to their esthetics and properties, both of which are highly dependent upon the microstructure of the materials. The type, size, amount, and distribution of the reinforcing fillers are all critical determinants of the optical and physical properties of the composite. The chemistry of the resin monomers and the quality of the highly cross-linked network formed during the polymerization reaction significantly influences these properties. Finally, the transfer of stress from the weaker resin matrix to the stronger and stiffer reinforcing fillers is accomplished by ensuring a strong interfacial linkage between the two phases, typically via a silane coupling agent. Recent work characterizing and describing the influence of the microstructure of dental composites on their properties and performance are reviewed in this manuscript.
\end{abstract}

Keywords Dental composite $\cdot$ Microstructure $\cdot$ Silane · Coupling agent $\cdot$ Monomer $\cdot$ Polymer network $\cdot$ Interface

\section{Introduction}

The microstructural features of dental composites exert a significant influence on the properties and performance of these materials. Dental composites fail primarily from the recurrence of dental decay around existing restorations and from fracture of the restorative material [1]. Both of these phenomena are likely related to the formulation of the

\author{
J. L. Ferracane $(\varangle) \cdot$ C. S. Pfeifer · T. J. Hilton \\ School of Dentistry, Oregon Health \& Science University, \\ 2730 S.W. Moody Ave, Portland, OR 97201, USA \\ e-mail: ferracan@ohsu.edu \\ C. S. Pfeifer \\ e-mail: pfeiferc@ohsu.edu \\ T. J. Hilton \\ e-mail: hiltont@ohsu.edu
}

materials, which influences factors such as shrinkage, bonding to tooth, resistance to cracking, and durability, and depend upon the composite microstructure.

The mechanical properties of dental composites are largely determined by the filler component, mainly because the properties of the reinforcing fillers are much higher than that of the polymer resin matrix. Dental composites have previously been shown to conform reasonably well to predictive models based on mechanics of complex viscoelastic structures, and not to a simple "rule of mixtures" relationship where the property is linearly related to the filler volume [2]. More recent studies confirm this dependence of mechanical properties on filler loading in commercial composites [3]. However, filler size, shape, and distribution are also important, especially for properties such as wear [4]. Further, the quality of the interfacial coupling between the reinforcing filler and the polymer resin matrix is critical for allowing the stress transfer between the matrix and filler, which deviates significantly from the ideal. The role of the polymer matrix also cannot be understated, because the chemical composition of the monomers, the extent of their polymerization, and the quality of the highly cross-linked network all significantly influence the physical properties of the final structure. Dental composites made from di-functional monomers with more rigid backbones and forming more highly converted and cross-linked networks are generally stronger and tougher than those made from more flexible monomers, or from compositions with lower conversion [5]. Thus, predicting the properties of the typical dental composite is complex and dependent upon characterizing each component of its microstructure. The following is a review of articles on the filler, matrix, and coupling agent, concentrating on those published in the past several years.

\section{Filler Phase}

A variety of ceramics and glasses have been used as fillers for dental composites. These materials are typically produced, or 
ground, to a sub-micrometer size for enhanced polish retention, and exist in spherical or irregular shapes. Recent studies have reported on the morphology of the fillers of different low viscosity dental composites and filled sealants, showing them to contain both spherical and irregularly shaped particles, depending upon brand, in a broad distribution of sizes $[6,7]$. The concept of incorporating a distribution of filler sizes, rather than a monomodal formulation, is consistent with the fact that reducing the inter-particle spacing, i.e., fitting smaller particles into spaces between larger particles, enhances the wear resistance, hardness, and strength of the composite, in part by minimizing the volume of the resin matrix [8]. The more highly filled materials are also more resistant to degradation of properties in solvents, such as ethanol [6].

Recently, one group reported on a comparison of the properties of composites with two different monomodal filler distributions, one being submicron in average size (approximately $400 \mathrm{~nm}$ but with all particles below $1 \mu \mathrm{m}$ ), and the other being micron sized (approximately $1000 \mathrm{~nm}$ average size but with all particles below $4 \mu \mathrm{m}$ ) [9]. The composites had similar radiopacity, strength, and creep resistance. The material with the submicron filler had lower elastic modulus and reduced degree of conversion (DC), but had higher hardness, higher gloss, and less surface roughness, and was able to maintain this smoother surface better than the larger particle composite after toothbrush abrasion.

\section{Surface Finish}

Large particles serve as good crack deflectors and enhance the strength of a composite. However, these particles also lead to higher surface roughness and reduced surface gloss [10], thus negatively affecting the optical properties of the composite. In fact, in a recent study using a series of different sized fillers of spherical $(100-1000 \mathrm{~nm})$ and irregular $(450-1500 \mathrm{~nm})$ shape to make dental composites with equivalent filler volume, surface roughness, as measured by Ra value, was reduced for composites with smaller fillers and spherical fillers [11]. Interestingly, in an unrelated study using composites made from these same fillers, it was shown that wear was also reduced with spherical fillers [4]. More recently, these same fillers were used to investigate the effect of filler size on void formation and fracture toughness of experimental composites [12]. The study showed that void formation was enhanced in composites with larger fillers (but the same filler volume), from a low of $0.27 \%$ voids for a composite with a trimodal distribution of irregular and spherical fillers to a high of $3.53 \%$ for a composite with a unimodal distribution of $1500-\mathrm{nm}$ irregular fillers. This outcome would seem to be supported by the concept of enhanced filler packing with smaller fillers or multimodal filler distributions. Though the study did not show a correlation between void formation and fracture toughness, the composite with the statistically highest fracture toughness had the trimodal filler distribution and the lowest void content. Having a high filler volume has also been shown to make it easier to create a high polish on composites [13].

\section{Degree of Cure and Shrinkage}

As the filler volume increases in the formulation of a dental composite, the amount of monomer available to react and produce shrinkage during curing is reduced. This relationship is essentially linear. This phenomenon has been reported many times, including very recently in a systematic study in which filler volume was varied and DC and shrinkage were measured [14].

Another recent study has evaluated the effect of the size of nanoparticles on the DC and mechanical properties of resin composites, showing DC to be reduced $(65.2 \%$ down to $59.5 \%$ ) as the particle size was increased from 7 to $40 \mathrm{~nm}$ [15]. The lower DC with larger particles in the nano-sized range was ascribed to enhanced light scattering, though there was little effect on mechanical properties.

Another study has shown a relationship between reduced polymerization shrinkage stress for composites containing spherical vs. irregular fillers of the same size and volume [16]. This result was consistent with the reduced shrinkage found for the same composites containing spherical fillers, and attributed to a likely reduced DC [17].

\section{Fracture, Toughness, and Fatigue Behavior}

The filler component has a great influence on the dental composite's ability to resist crack initiation and propagation, two critical determinants of fracture, as well as its response to abrasion and contact loading leading to wear. Because these properties influence the overall clinical performance of dental composites, it is important to better elucidate the manner in which the filler microstructure influences important properties, such as fracture, toughness, and fatigue.

A recent study of dynamic fatigue using biaxial flexure testing revealed that composites with a broader distribution of filler particle sizes had a higher resistance to subcritical crack growth and therefore an enhanced in vitro lifetime [18]. Theoretically, this would result in an enhanced clinical longevity via greater resistance to crack growth in restorations that ultimately leads to catastrophic fracture. However, in a follow-up study, this group examined the fracture toughness, initial fracture strength, and cyclic fatigue resistance of the same composites, and showed that the presence of larger particles led to enhanced fracture toughness owing to greater crack deflection energy-dissipating mechanisms [19••]. 
However, composites with smaller fillers had improved fatigue resistance owing to the fact that they were more creep compliant, thus allowing them to dissipate lower load cyclic energy more efficiently. Thus, composites with larger fillers would be expected to be more resistant to the rapid introduction of high contact forces, such as when teeth impact on one another or the patient bites down rapidly on a hard object, while those with smaller fillers would be superior under the conditions of lower cyclic stresses, such as normal chewing.

Current evidence seems to point in the direction of lower mechanical properties for most nanohybrid composites [20]. These materials typically incorporate pre-polymerized resin fillers (PPRF) that reduce overall monomer content to reduce shrinkage, but are not as strong as solid glass-reinforcing fillers. Composites with PPRF do polish well, and in fact have been shown to produce composites that retain smoother surfaces after abrasion than commercial hybrid composites containing a high concentration of irregular-shaped fillers [21]. In contrast, the incorporation of clusters of nano-sized inorganic particles in a commercial composite does not negatively affect mechanical properties, in large part because of the fact that high filler loadings can be accomplished and the particle clusters serve as efficient reinforcing agents [22-24].

Recent studies have reported on composites with enhanced fracture toughness due to the incorporation of other types of fillers, such as ceramic nanofibers [25] and hydroxyapatite whiskers [26]. These particles provide toughening through enhanced energy-dissipating events during crack propagation, such as crack deflection, bridging, pinning, and fiber pullout. These toughening mechanisms have previously been identified for dental composites containing nano- and micro-sized fillers as well [22].

\section{Resin Phase}

While most of the improvements made to composites throughout the years have involved the filler technology, more recently, modifications on the chemistry of monomers have been proposed in an attempt to address some of the challenges associated with these materials. One of the main drawbacks with the use of composites is still the inherent volumetric shrinkage associated with the polymerization of methacrylates. Because this shrinkage takes place under confinement owing to the adhesion to the walls of the preparation, stress is transferred to the bonded interface, which in turn can damage the margins by causing discoloration, leakage, and eventually secondary decay. Stress results from a complex interaction between shrinkage, conversion, and elastic modulus development [27•]. Because high conversion and high modulus are always desired, the efforts to reduce stress have focused on reducing shrinkage or directly reducing stress [27•].
Apart from shrinkage and polymerization stress, another issue correlated to the resin matrix is the degradation of the material by hydrolysis and enzymatic attack of the methacrylate ester bonds over time [28], which is especially problematic because the DC of commercially available composites rarely surpasses 65-70\% [1]. The aging process not only weakens the restoration, but also jeopardizes esthetic parameters such as loss of gloss, staining, and increased surface roughness [29, 30]. Each of these issues, along with more recent attempts to resolve them, will be discussed in the subsequent sections.

\section{Volumetric Shrinkage and Shrinkage Stress}

Conventional composites based on bisphenol A-glycidyl methacrylate (BisGMA) usually require the addition of a low viscosity diluent to allow for the inclusion of a larger concentration of fillers and to improve DC. However, the increase in DC and the fact that such diluents, in general, are also of low molecular weight, lead to higher volumetric shrinkage and shrinkage stress [31]. Fairly recently, manufacturers have concentrated on a series of strategies to reduce shrinkage and shrinkage stress, including the development of highmolecular-weight monomers. Commercial examples include the Dimer dicarbamate dimethacrylate, present in N'Durance ${ }^{\mathrm{TM}}$ and DX-511, a technology developed by Dupont ${ }^{\mathrm{TM}}$, used in

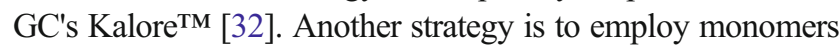
with an intrinsically lower, molar shrinkage coefficient, such as those that polymerize through a ring-opening mechanism. In simple terms, the polymerization of these monomers results in less shrinkage per molecule engaging in network formation. To date, one commercial material based on oxirane chemistry has been released [33], which presents on average $50 \%$ less shrinkage and reduced stress, but mechanical properties comparable to that of methacrylate counterparts [32, 34]. Since then, spiro-ortho carbonates [35, 36] and other ring-opening monomers [37] have been designed with that aim. Along the same lines, liquid crystalline, bisphenyl epoxy resins have also been described for use as dental composites, with the advantage of increasing hardness and flexural modulus and decreasing water sorption and coefficient of thermal expansion, as well as inducing phase separation [38]. Apart from reduced shrinkage, one additional advantage of the epoxy resins is their hydrophobicity, which could contribute to decreased water sorption/ solubility [32, 34]. However, challenges associated with cationic polymerizations, especially in a moist environment such as the oral cavity, make the restorative procedure even more technique sensitive. Clinical studies using commercially available materials tend to agree that conventional dimethacrylate resins and low-shrinkage products behave similarly in terms of marginal degradation [39, 40]. Even in vitro studies have demonstrated that not all materials marketed as "low shrinkage" lead to lower 
stress values, likely owing to the complex relationship between DC, volumetric shrinkage, and elastic modulus [41-43].

Another way of reducing the shrinkage is the inclusion of pre-polymerized additives. Similar to higher molecular weight monomers, this approach reduces the concentration of functional groups available to react per unit volume of material. Though the idea is not necessarily new, more recently nonfunctionalized [44] and functionalized pre-polymerized particles have been described [45-47]. Star-shaped photocurable poly(methyl methacrylate) macromonomers have been shown to increase conversion, improve the potential for crosslinking, and reduce shrinkage [44]. Additionally, nanogels have been used to successfully reduce shrinkage and stress in dimethacrylate networks, while keeping mechanical properties similar to the controls [45]. The advantage of this type of additive is that the structure can be designed to target specific properties (refractive index, toughness, hydrophilicity) and also to include polymerizable functionalities and/or photoinitiator molecules to locally control polymerization rate $[48,49]$. Polyhedral oligomeric silsesquioxanes (POSS) have also been described for use as dental materials [50, 51]. POSS is an organic-inorganic hybrid nanoparticle consisting of a silica cage core, as well as other organic groups attached to the corners of the cage. The addition of small amounts of POSS leads to material reinforcement and increased resistance to wear [50].

While reducing polymerization shrinkage is a good approach to reducing stress, more recently, efforts have concentrated on directly reducing polymerization stress. As the polymerization reaction progresses for multifunctional monomers, network development gradually imposes limitations to diffusion of reactive species, which become trapped in a crosslinked network. In this transition from a viscous to a viscoelastic state, the onset of a significant increase in elastic modulus (vitrification stage) coincides with the onset of appreciable stress development [52]. One approach to reduce stress is to manipulate polymerization reaction conditions to delay gelation/vitrification. This can be accomplished with the use of thiol-enes or thiol-methacrylate formulations, in which chain-transfer reactions of the thiol to the vinyl effectively work to delay the point in conversion at which stiffness starts to develop, hence delaying and significantly reducing polymerization stress [27•, 53, 54]. By judiciously choosing the comonomer systems, these materials can also present mechanical properties comparable to the conventional dimethacrylate materials, but with higher conversion and lower stress [54, 55]. Drawbacks with this technology that have delayed its commercial implementation include the foul odor and stability concerns (shelf-life) associated with thiol-containing materials. As alternatives, thiol-terminated oligomeric additives have been proposed, with increased mechanical properties, increased conversion, and decreased stress [53].

The use of polymerization-induced phase-separation (PIPS) has also been advocated as a way to accommodate shrinkage development during polymerization [56]. PIPS leads to the formation of a multi-phase polymer structure with heterogeneous domains distributed co-continuously or in droplet-matrix fashion [57, 58]. By tailoring the monomer systems (or additives) it is possible to combine desirable properties of different networks, as well as create opportunities for shrinkage and stress relaxation at interfacial regions between the domains [57, 58]. For example, the use of liquid crystals has been recently proposed as one way to effectively drive phase separation in dental materials $[59,60]$.

Another more recent approach is the use of covalently adaptable networks, capable of undergoing stress relaxation via addition-fragmentation chain transfer [61, 62]. This resin system contains allyl sulfide functional groups, which enable stress relaxation within the polymerizing network through rearrangement of its connectivity without reducing crosslinking density or ultimately mechanical properties. In other words, this is a non-degradative process of chain fragmentation and recombination, and allows the material to retain its physico-chemical and mechanical properties before and after the mechanism is initiated $[61,62]$.

\section{Incomplete DC, Depth of Cure, and Material Degradation}

The polymerization reaction in restorative composites is photoinitiated with visible light and relies on deep light penetration to assure the achievement and maintenance of good mechanical properties. Barriers to light penetration include scattering by the filler particles and the inner shielding effect of camphorquinone (the photo-initiator acts as a filter to specific wavelengths). For most commercial composites, this means the material needs to be placed in relatively thin increments, increasing placement time and the sensitivity of the restorative technique. Recently, a series of bulk-fill composites were introduced, claiming to be curable in 4- to $5-\mathrm{mm}$ increments using the same exposure time/intensity recommended for conventional composites [63]. This is in theory made possible by either a reduction in the filler content (many are flowable composites), improving the translucency of the material by altering the filler/matrix composition, or by changing the photoinitiator system $[64,65]$. One manufacturer has developed a germanium-based photoinitiator (Ivocerin ${ }^{\mathrm{TM}}$ ) claiming improved depth of cure in regular consistency composites. To date, in vitro studies have demonstrated that, in general, mechanical properties (flexural strength/modulus and microhardness) and conversion tend to be similar or lower for bulk-fill materials, even when polymerized in 2-mm increments [65]. However, the depth of cure assessed with the International Standards Organization (ISO) standard is greater for bulk-fill materials [66, 67], attributed to their increased translucency [63].

Independent of the restorative technique, a good depth of cure is necessary to ensure adequate mechanical properties, as well as to minimize the potential for degradation of the resin 
matrix and bonded interface accompanying poorly cured materials [68]. Leachable components from conventional materials include the actual monomers and some of their degradation products, such as triethylene glycol and bishydroxypropoxy-phenyl propane $[69,70]$. These products have been demonstrated to regulate bacterial adhesion and colonization $[71,72]$, as well as to pose potential toxicity concerns, especially when used in dental adhesives [72-75]. Several direct restorative materials are in fact conducive of biofilm colonization, and therefore, antimicrobial composites have also been proposed $[76,77]$. The most common approaches are based either on charged sites within the monomer (e.g., quaternary alkylammonium functionalities) [76, 78], or the addition of triclosan monomer units [77] or Irgasan-containing delivery beads [79]. While general mechanical properties and polymerization efficiency remain unaltered in comparison to conventional counterparts, the biological activity and substantivity of such materials remain to be elucidated.

Apart from biofilm-related concerns, the potential for degradation of methacrylate-based composites and adhesives by hydrolysis and enzymatic attack has prompted the development of methacrylamide-based materials [80, 81]. Some of these materials produce less leachable components when stored in water or organic solutions [81], and in the case of tertiary methacrylamides, have also demonstrated resistance to esterase degradation [83]. This is important to preserve mechanical and optical properties, as well as reduce the amount of leachates, including the potential for bisphenol A (BPA) release in BisGMA-based materials [83].

\section{Coupling Agents}

Coupling agents are used to chemically adhere the inorganic filler to the organic resin matrix, as well as to enhance particle wetting so as to maximize filler loading in the resultant composite [27•]. Both the chemical adhesion of filler to resin and increased filler load improves resin composite mechanical properties and clinical performance. The most commonly used coupling agent for dental resin composites is 3methacryloxypropyltrimethoxysilane (MPS). However, composites incorporating fillers bonded with MPS are subject to chemical degradation over time because of water sorption and hydrolysis of the fillers. As a result, researchers have investigated alternative composite formulations, either using alternative coupling agents or an alternative filler that precludes the necessity for a coupling agent [27•].

Itota et al. theorized that a coupling agent with greater hydrophilicity and alkalinity compared with 3-MPS would increase fluoride release and alkalinity, resulting in decreased recurrent caries [84]. To test this hypothesis, the authors fabricated experimental composites in which a fluoroboroaluminosilicate glass filler was silanated with
3-MPS or 3-aminopropyltrimethoxysilane (3-AS). A composite with non-silanated filler particles was also tested. The composite made with 3-AS showed significantly increased water sorption, fluoride release, and $\mathrm{pH}$ compared with the other two groups. However, the $\mathrm{pH}$ neutralizing effect decreased with time and there was no significant difference among the groups in artificial caries lesion formation. There was no evaluation of the effect of increased water sorption on mechanical properties.

Another group evaluated the effect of altering filler particle structure to eliminate the use of a coupling agent altogether. They fabricated a nano-structured resin composite made up of anodic porous alumina that used mechanical interlocking of the porous filler particle with the resin matrix in lieu of using a coupling agent. The storage modulus of the composite made with porous particles was higher than that of the non-porous counterpart and comparable to two commercial nanofilled composites. Artificial aging resulted in less degradation in the storage modulus of the porous particle composite compared with the commercial composites, although at higher testing temperatures, the absolute values were similar [85•].

\section{Conclusions}

The properties of dental composites are highly dependent upon the quality and concentration of the reinforcing fillers, the polymer network forming monomers, and the coupling agent that links the two to support stress transfer from the weaker polymer to the stronger filler. Recent studies have investigated the effect of reinforcing particle size and shape, as well as interfacial modifications, on the performance of dental composites. However, improvements are limited, and current materials still do not have adequate strength to resist intra-oral fracture like more high-strength materials. Significant developments have taken place in producing new monomers for the resin matrix binder, focusing on reducing shrinkage and its accompanying stress, and making more degradationresistant materials. While current materials are of very high quality, improvements are needed, a fact that fuels intensive research and development.

\section{Compliance with Ethics Guidelines}

Conflict of Interest Dr. Jack L. Ferracane is a board member for Blue Light Analytics. Drs. Ferracane, Pfeifer and Hilton have received grants from NIH/NIDCR, various honoraria and royalties from book sales, and declare that many manufacturers support research and product testing in their laboratory on a fee for service or research grant basis, always via a contract negotiated with the Oregon Health \& Science University.

Human and Animal Rights and Informed Consent This article does not contain any studies with human or animal subjects performed by any of the authors. 


\section{References}

Papers of particular interest, published recently, have been highlighted as:

- Of importance

-. Of major importance

1. Ferracane JL. Resin-based composite performance: are there some things we can't predict? Dent Mater. 2013;29(1):51-8.

2. Chantler PM, Hu X, Boyd NM. An extension of a phenomenological model for dental composites. Dent Mater. 1999;15(2):144-9.

3. Jun SK, Kim DA, Goo HJ, et al. Investigation of the correlation between the different mechanical properties of resin composites. Dent Mater J. 2013;32(1):48-57.

4. Turssi CP, Ferracane JL, Vogel K. Filler features and their effects on wear and degree of conversion of particulate dental resin composites. Biomaterials. 2005;26(24):4932-7.

5. Musanje L, Ferracane JL. Effects of resin formulation and nanofiller surface treatment on the properties of experimental hybrid resin composite. Biomaterials. 2004;25(18):4065-71.

6. Borges $\mathrm{BC}$ et al. Filler morphology of resin-based low-viscosity materials and surface properties after several photoactivation times. Acta Odontol Scand. 2013;71(1):215-22.

7. Salerno M et al. Surface morphology and mechanical properties of new-generation flowable resin composites for dental restoration. Dent Mater. 2011;27(12):1221-8.

8. Lim BS et al. Effect of filler fraction and filler surface treatment on wear of microfilled composites. Dent Mater. 2002;18(1):1-11.

9. Valente LL et al. Comparative evaluation of dental resin composites based on micron- and submicron-sized monomodal glass filler particles. Dent Mater. 2013;29(11):1182-7.

10. da Costa $J$ et al. The effect of various dentifrices on surface roughness and gloss of resin composites. J Dent. 2010;38 Suppl 2:e123-128.

11. Marghalani HY. Effect of filler particles on surface roughness of experimental composite series. J Appl Oral Sci. 2010;18(1): $59-67$.

12. Elbishari H, Silikas N, Satterthwaite J. Filler size of resin-composites, percentage of voids and fracture toughness: is there a correlation? Dent Mater J. 2012;31(4):523-7.

13. Jasse FF et al. Influence of filler charge on gloss of composite materials before and after in vitro toothbrushing. J Dent. 2013;41 Suppl 5:e41-44.

14. Shah PK, Stansbury JW. Role of filler and functional group conversion in the evolution of properties in polymeric dental restoratives. Dent Mater. 2014;30(5):586-93.

15. Karabela MM, Sideridou ID. Synthesis and study of properties of dental resin composites with different nanosilica particles size. Dent Mater. 2011;27(8):825-35.

16. Satterthwaite JD et al. Effect of resin-composite filler particle size and shape on shrinkage-stress. Dent Mater. 2012;28(6):609-14.

17. Satterthwaite JD, Vogel K, Watts DC. Effect of resin-composite filler particle size and shape on shrinkage-strain. Dent Mater. 2009;25(12):1612-5.

18. Ornaghi BP et al. Subcritical crack growth and in vitro lifetime prediction of resin composites with different filler distributions. Dent Mater. 2012;28(9):985-95.

19.• Ornaghi BP et al. Fracture toughness and cyclic fatigue resistance of resin composites with different filler size distributions. Dent Mater. 2014;30(7):742-51. The study describes the effect of filler particle size distribution on dental composite properties. It shows how the development of high fracture toughness, optimally achieved through the addition of large particles, may not always result in the most fatigue resistant composite, which seems to be more dependent upon having smaller fillers that allow more efficient dissipation of energy during cyclic loading.

20. Blackham JT, Vandewalle KS, Lien W. Properties of hybrid resin composite systems containing prepolymerized filler particles. Oper Dent. 2009;34(6):697-702.

21. Kakuta $\mathrm{K}$ et al. Surface textures of composite resins after combined wear test simulating both occlusal wear and brushing wear. Dent Mater J. 2012;31(1):61-7.

22. Shah MB, Ferracane JL, Kruzic JJ. R-curve behavior and micromechanisms of fracture in resin based dental restorative composites. J Mech Behav Biomed Mater. 2009;2(5):502-11.

23. Shah MB, Ferracane JL, Kruzic JJ. Mechanistic aspects of fatigue crack growth behavior in resin based dental restorative composites. Dent Mater. 2009;25(7):909-16.

24. Rodrigues Jr SA et al. Microstructural characterization and fracture behavior of a microhybrid and a nanofill composite. Dent Mater 2008;24(9):1281-8.

25. Guo $\mathrm{G}$ et al. Novel dental composites reinforced with zirconia-silica ceramic nanofibers. Dent Mater. 2012;28(4):360-8.

26. Zhang H, Darvell BW. Mechanical properties of hydroxyapatite whisker-reinforced bis-GMA-based resin composites. Dent Mater. 2012;28(8):824-30.

27. Cramer NB, Stansbury JW, Bowman CN. Recent advances and developments in composite dental restorative materials. J Dent Res. 2011;90(4):402-16. This comprehensive review summarizes the advances in composite materials until 2011, as well as providing background information on mechanisms of polymerization, stress development, and mechanical properties driving the development of new materials.

28. Cai K, et al. Biodegradation of composite resin with ester linkages: Identifying human salivary enzyme activity with a potential role in the esterolytic process. Dent Mater. 2014. doi:10.1016/j.dental. 2014.05.031.

29. Catelan A et al. Color stability of sealed composite resin restorative materials after ultraviolet artificial aging and immersion in staining solutions. J Prosthet Dent. 2011;105(4):236-41.

30. Anfe TEDA, Agra CM, Vieira GF. Evaluation of the possibility of removing staining by repolishing composite resins submitted to artificial aging. J Esthet Rest Dent. 2011;23(4):260-7.

31. de Godoy Fróes-Salgado NR, et al. Influence of the base and diluent monomer on network characteristics and mechanical properties of neat resin and composite materials. Odontology. 2014. doi:10.1007/ s10266-014-0153-6.

32. Boaro LC et al. Sorption, solubility, shrinkage and mechanical properties of "low-shrinkage" commercial resin composites. Dent Mater. 2013;29(4):398-404.

33. Eick JD et al. Properties of silorane-based dental resins and composites containing a stress-reducing monomer. Dent Mater. 2007;23(8):1011-7.

34. Yamasaki LC et al. Polymerization development of "low-shrink" resin composites: reaction kinetics, polymerization stress and quality of network. Dent Mater. 2013;29(9):e169-79.

35. Sun $\mathrm{X}$ et al. Shrinkage properties of a modified dental resin composites containing a novel spiro-orthocarbonate expanding monomer. Materials Lett. 2011;65(23-24):3586-9.

36. $\mathrm{Fu} \mathrm{J}$ et al. Characterization of a low shrinkage dental composite containing bismethylene spiroorthocarbonate expanding monomer. Int J Molec Sci. 2014;15(2):2400-12.

37. Vitale A et al. Visible light curable restorative composites for dental applications based on epoxy monomer. Materials. 2014;7(1):554-62.

38. Hsu SH et al. Biphenyl liquid crystalline epoxy resin as a lowshrinkage resin-based dental restorative nanocomposite. Acta Biomater. 2012;8(11):4151-61.

39. Baracco B et al. Two-year clinical performance of a low-shrinkage composite in posterior restorations. Oper Dent. 2013;38(6): 591-600. 
40. Baracco B et al. Clinical evaluation of a low-shrinkage composite in posterior restorations: one-year results. Oper Dent. 2012;37(2): $117-29$.

41. Watts DC, Alnazzawi A. Temperature-dependent polymerization shrinkage stress kinetics of resin-composites. Dent Mater. 2014;30(6):654-60.

42. Frauscher KE, Ilie N. Depth of cure and mechanical properties of nano-hybrid resin-based composites with novel and conventional matrix formulation. Clin Oral Invest. 2012;16(5):1425-34.

43. Frauscher KE, Ilie N. Degree of conversion of nano-hybrid resinbased composites with novel and conventional matrix formulation. Clin Oral Invest. 2013;17(2):635-42.

44. Szanka A, Szarka G, Iván B. Multi-methacrylated star-shaped, photocurable poly(methyl methacrylate) macromonomers via quasiliving ATRP with suppressed curing shrinkage. Polymer (United Kingdom). 2013;54(22):6073-7.

45. Moraes RR et al. Control of polymerization shrinkage and stress in nanogel-modified monomer and composite materials. Dent Mater. 2011;27(6):509-19.

46. Liu J et al. A study of shrinkage stress reduction and mechanical properties of nanogel-modified resin systems. European Polymer J. 2012;48(11):1819-28.

47. Szaloki M et al. Synthesis and characterization of cross-linked polymeric nanoparticles and their composites for reinforcement of photocurable dental resin. Reactive Functional Polymers. 2013;73(3):465-73.

48. Dailing E et al. Nanogels as a basis for network construction. Macromol Symp. 2013;329(1):113-7.

49. Liu J et al. Photo-reactive nanogels as a means to tune properties during polymer network formation. Polymer Chem. 2014;5(1): 227-33.

50. Wang W et al. Structure-property relationships in hybrid dental nanocomposite resins containing monofunctional and multifunctional polyhedral oligomeric silsesquioxanes. Int J Nanomedicine. 2014;9(1):841-52.

51. Bredov NS et al. Synthesis of oligoorganosilsesquioxanes via acidohydrolytic polycondensation. Polymer Sci - Series B. 2013;55(7-8):472-7.

52. Pfeifer $\mathrm{CS}$ et al. Delayed gelation through chain-transfer reactions: mechanism for stress reduction in methacrylate networks. Polymer. 2011;52(15):3295-303.

53. Reinelt $\mathrm{S}$, et al. Synthesis and photopolymerization of thiolmodified triazine-based monomers and oligomers for the use in thiol-ene-based dental composites. Macromolec Chem Phys. 2014. doi:10.1002/macp.201400174.

54. Beigi S, Yeganeh H, Atai M. Evaluation of fracture toughness and mechanical properties of ternary thiol-ene-methacrylate systems as resin matrix for dental restorative composites. Dent Mater. 2013;29(7):777-87.

55. Boulden JE et al. Thiol-ene-methacrylate composites as dental restorative materials. Dent Mater. 2011;27(3):267-72.

56. Bracho-Troconis $\mathrm{C}$ et al. Characterization of N'Durance: a nanohybrid composite based on new nano-dimer technology. Comp Contin Educ Dent. 2010;31(Spec No 2):5-9.

57. Pfeifer $\mathrm{CS}$ et al. Tailoring heterogeneous polymer networks through polymerization-induced phase separation: influence of composition and processing conditions on reaction kinetics and optical properties. J Polymer Sci, Part A: Polymer Chemistry. 2014;52(13): 1796-806

58. Szczepanski CR, Pfeifer CS, Stansbury JW. A new approach to network heterogeneity: polymerization induced phase separation in photo-initiated, free-radical methacrylic systems. Polymer (United Kingdom). 2012;53(21):4694-701.

59. Liu W et al. Synthesis of a liquid-crystalline resin monomer with the property of low shrinkage polymerization. Dent Mater J. 2013;32(4):550-6.
60. Liu WW et al. Investigation of the mechanical properties of a lowshrinkage liquid crystalline matrix combined with nano-hydroxyapatite. Int J Nanomedicine. 2011;6:1787-91.

61. Park HY et al. Novel dental restorative materials having low polymerization shrinkage stress via stress relaxation by additionfragmentation chain transfer. Dent Mater. 2012;28(11):1113-9.

62. Park $\mathrm{HY}$ et al. Stress relaxation via addition-fragmentation chain transfer in high $\mathrm{T} g$, high conversion methacrylate-based systems. Macromolecules. 2012;45(14):5640-6.

63. Bucuta S, Ilie N. Light transmittance and micro-mechanical properties of bulk fill vs. conventional resin based composites. Clin Oral Investig. 2014. doi:10.1007/s00784-013-1177-y.

64. Leprince JG et al. Physico-mechanical characteristics of commercially available bulk-fill composites. J Dent. 2014;42(8):993-1000.

65. Ilie N, Bucuta S, Draenert M. Bulk-fill resin-based composites: an in vitro assessment of their mechanical performance. Oper Dent. 2013;38(6):618-25.

66. Alrahlah A, Silikas N, Watts DC. Post-cure depth of cure of bulk fill dental resin-composites. Dent Mater. 2014;30(2):149-54.

67. Flury S et al. Depth of cure of resin composites: is the ISO 4049 method suitable for bulk fill materials? Dent Mater. 2012;28(5): $521-8$.

68. Lempel E, et al. Quantification of conversion degree and monomer elution from dental composite using HPLC and micro-Raman spectroscopy. Chromatographia. 2014. doi:10.1007/s10337-0142647-3.

69. Gul P, Miloglu FD, Akgul N. HPLC analysis of eluted monomers from dental composite using different immersion media. J Liquid Chromat Related Tech. 2014;37(2):155-70.

70. Bourbia $\mathrm{M}$ et al. Cariogenic bacteria degrade dental resin composites and adhesives. J Dent Res. 2013;92(11):989-94.

71. Velazquez-Enriquez $U$ et al. Quantitative analysis of $\mathrm{S}$. mutans and S. sobrinus cultivated independently and adhered to polished orthodontic composite resins. J Applied Oral Sci. 2012;20(5):544-9.

72. Schweikl H et al. 2-Hydroxyethyl methacrylate-induced apoptosis through the ATM- and p53-dependent intrinsic mitochondrial pathway. Biomaterials. 2014;35(9):2890-904.

73. Mathisen GH, et al. TEGDMA and filler particles from dental composites additively attenuate LPS-induced cytokine release from the macrophage cell line RAW 264.7. Clin Oral Invest. 2014. doi: 10.1007/s00784-014-1212-7.

74. Moilanen LH, Dahms JK, Hoberman AM. Reproductive toxicity evaluation of the dental resin monomer bisphenol a glycidyl methacrylate (CAS 1565-94-2) in mice. Int $\mathrm{J}$ Toxicol. 2013;32(6):415-25.

75. Moilanen LH, Dahms JK, Hoberman AM. Reproductive toxicity evaluation of the dental resin monomer triethylene glycol dimethacrylate (CASRN 109-16-0) in mice. Int J Toxicol. 2014;33(2):106-15.

76. Buruiana $\mathrm{T}$ et al. New urethane oligodimethacrylates with quaternary alkylammonium for formulating dental composites. J Mater Sci Mater Med. 2014;25(4):1183-94.

77. Melinte V et al. Photopolymerizable phosphate acrylates as comonomers in dental adhesives with or without triclosan monomer units. Mater Sci Eng C. 2014;34(1):176-85.

78. Zhou $\mathrm{C}$ et al. Synthesis of new antibacterial quaternary ammonium monomer for incorporation into $\mathrm{CaP}$ nanocomposite. Dent Mater. 2013;29(8):859-70.

79. Rüttermann $\mathrm{S}$, et al. Bacterial viability and physical properties of antibacterially modified experimental dental resin composites. PLoS ONE. 2013. doi:10.1371/journal.pone.0079119.

80. Catel Y, Fischer UK, Moszner N. Monomers for adhesive polymers: 11. Structure-adhesive properties relationships of new hydrolytically stable acidic monomers. Polymer Int. 2013;62(12):1717-28. 
81. Moszner N et al. Monomers for adhesive polymers, 9-synthesis, radical photopolymerization, and properties of (Meth)acrylamido dihydrogen phosphates. Macromolecular Mater Eng. 2013;298(4):454-61.

82. Pan $\mathrm{H}$ et al. Backbone degradable multiblock N-(2hydroxypropyl)methacrylamide copolymer conjugates via reversible addition-fragmentation chain transfer polymerization and thiolene coupling reaction. Biomacromolecules. 2011;12(1):247-52.

83. Lizenboim $\mathrm{K}$ et al. Bisphenol-A free dental polymeric materials. J Adhesion Sci Tech. 2013;27(4):354-70.
84. Itota $T$ et al. Neutralizing effect by resin-based materials containing silane-coated glass fillers. Dent Mater J. 2010;29(4):362-8.

85. Thorat SB, Diaspro A, Salerno M. In vitro investigation of coupling-agent-free dental restorative composite based on nanoporous alumina fillers. J Dent. 2014;42(3):279-86. This article demonstrated that it may be possible to couple fillers to the resin matrix via mechanical interlocking, thereby eliminating the need for a chemical coupling agent. If viable, this technique would help to limit property degradation over time. 